\title{
An Adaptive PID Neural Network for Complex Nonlinear System Control
}

\author{
Jun Kanga,b, Wenjun Meng ${ }^{\mathrm{a}}$, Ajith Abraham ${ }^{\mathrm{c}, \mathrm{d}, \mathrm{e}}$, Hongbo Liu ${ }^{\mathrm{c}, \mathrm{e}, *}$ \\ ${ }^{a}$ School of Mechanical Engineering, Taiyuan University of Science and Technology, \\ Taiyuan 030024, China \\ ${ }^{b}$ Software School, North University of China, Taiyuan 030051, China \\ ${ }^{c}$ Machine Intelligence Research Labs (MIR Labs), Scientific Network for Innovation and \\ Research Excellence, Auburn 98071, WA, USA \\ ${ }^{d}$ IT4Innovations - Center of excellence, VSB - Technical University of Ostrava, Ostrava \\ 70833, Czech Republic \\ ${ }^{e}$ School of Information Science and Technology, Dalian Maritime University, Dalian \\ 116026, China
}

\begin{abstract}
Usually it is difficult to solve the control problem of a complex nonlinear system. In this paper, we present an effective control method based on adaptive PID neural network and particle swarm optimization (PSO) algorithm. PSO algorithm is introduced to initialize the neural network for improving the convergent speed and avoiding weights getting trapped into local optima. To adapt the initially uncertain and varying parameters in the control system, we introduce an improved gradient descent method to adjust the network parameters. The stability of our controller is analyzed according to the Lyapunov method. The simulation of complex nonlinear multiple-input and multiple-output (MIMO) system is presented with strong coupling. Empirical results illustrate that the proposed controller can obtain good precision with shorter time compared with the other considered methods. It provides a novel control approach for complex nonlinear systems.
\end{abstract}

Keywords: Complex Nonlinear System, Adaptive, PID Neural Network, PSO, Gradient Descent

\footnotetext{
${ }^{*}$ Corresponding author.

Email addresses: kj_ty0015@sina.com (Jun Kang), tyustmwj@126.com (Wenjun Meng), ajith.abraham@ieee.org (Ajith Abraham), lhb@dlut.edu.cn (Hongbo Liu)
} 


\section{Introduction}

In the industrial control field, the controlled system usually has great nonlinearity, including spacecraft system, vehicle system, robot system, power system, chemical reaction system, etc. PID control technique has been widely used in the real control system for its advantages such as simple mechanism and clear physical conception. It is hard to get a precise control performance even while researchers focus on other intelligent control methods, including adaptive control [1, 2], fuzzy control [3, 4, 5], neural network control [6, 17, 8] and decoupling control [9, 10, 11] etc.. And then, some mixed control methods are emerging, such as PID neural network. Due to the characteristics of self-learning, self-organizing and self-adaptation, PID neural network would automatically identify the controlled system parameters and automatically adjust the parameters according to the changes in the process parameters.

In this paper, we design a controller model based on an adaptive PID neural network. To prevent the weights of neural network falling into local optima, PSO algorithm is adopted to select initial weights. The parameters of PID neural network are self-regulating without intervention. The improved gradient descent method is used to optimize the weights of network.

\section{Related Works}

Since it is difficult to control a complex nonlinear system [12, 13, 14], neural network was introduced to solve the problem [15, 16, 17]. However, researches still confront some difficulties. For example, network parameters training is time-consuming and easily falls into local minimum. Particle swarm optimization (PSO) algorithm is a new globe optimization algorithm, which has the advantage of fast convergence speed [18, 19]. In [20], Selvakumaran et al. proposed a new design of decentralized load-frequency controller for interconnected power systems with ac-dc parallel using PSO algorithm. The experiment result illustrated that their method have rapid dynamic response ability. In [21], Hasni et al. used PSO algorithm to parameters selection, and used genetic algorithm (GA) to optimize the choice of parameters by minimizing a cost function. The study result was applied to a greenhouse environment with Continuous Roof Vents, and obtained satisfactory effect. In [22], Chen et al. designed a novel multi-objective endocrine particle swarm optimization algorithm. The method is equipped with the advantages of some multi-objective optimization problems. Nevertheless, these 


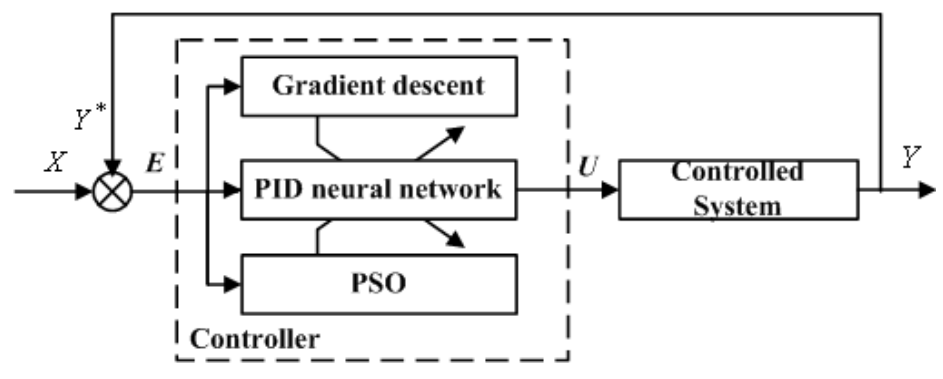

Figure 1: Structure of control system.

methods mentioned above can not be applied to complex nonlinear system with strong coupling.

Adaptive controller has the ability to adjust of control parameters without the help of human intelligence. It can tune complex systems better by combining nonlinear controlling methods and intelligent control technology [23, 24]. The results show that adaptive control has the advantage to solve effectively problems of nonlinear system with uncertain model and random disturbance.

\section{Adaptive PID Neural Networks}

\subsection{Control system structure}

The control system adopts close loop control, and it mainly consists of two parts: the controller and the controlled system, as shown in Figure 1. The controller is built based on adaptive PID neural network. In the whole control system, $X$ is object vector, $E$ is error vector, $Y$ is output value of control system. And $U$ is control law of the control system. The controlling algorithm is illustrated in Algorithm 1.

\subsection{PID neural network controller}

In the controller, three-layer PID neural network is built by combining PID and feedforward neural network, as shown in Figure 2, $X^{*}=\left[\begin{array}{ll}X Y & \text { is }\end{array}\right.$ input vector of the controller, $X=\left[x_{1}, x_{2}, \cdots, x_{n}\right]^{T}$ is object value of the whole control system, and $Y=\left[y_{1}^{*}, y_{2}^{*}, \cdots, y_{n}^{*}\right]^{T}$ is a feedback value from current system output.

Input layer have $2 n$ neurons, $n$ of them are used to input object values, the others are used to input values which returned from control system's output. The output of this layer at $k$ is 

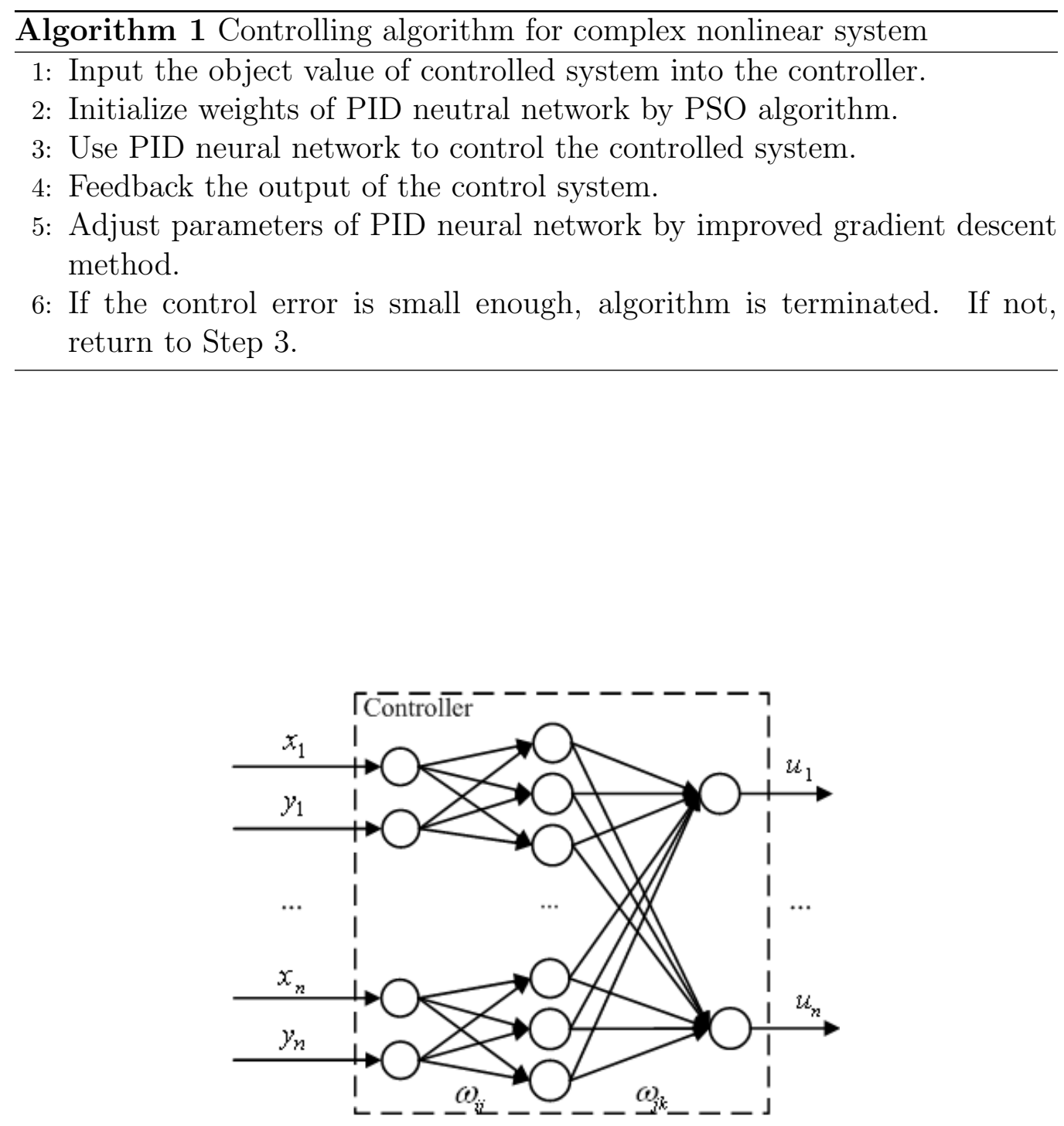

Figure 2: Structure of PID neural network 


$$
\begin{aligned}
& \text { out }_{q 1}^{1}(k)=x_{q}(k) \\
& \text { out }_{q 2}^{1}(k)=y_{q}^{*}(k)
\end{aligned}
$$

Hidden layer have $3 n$ neurons, including $n$ proportion neurons, $n$ integration neurons and $n$ differentiation neurons. The output of each neuron in this layer at $k$ is

$$
\begin{gathered}
\text { out }_{q 2}^{2}(k)=\phi_{p} \sum_{i=1}^{2} \omega_{i 1} \text { out } \\
q i \\
\text { out }_{q 2}^{2}(k)=\phi_{i}\left[\sum_{i=1}^{2} \omega_{i 2}(k) x_{l i}(k)+o u t_{q 2}^{2}(k-1)\right] \\
\text { out }_{q 3}^{2}(k)=\phi_{d}\left[\sum_{i=1}^{2} \omega_{i 3}(k) x_{l i}(k)-\sum_{i=1}^{2} \omega_{i 3}(k-1) x_{l i}(k-1)\right]
\end{gathered}
$$

where, $\phi_{p}, \phi_{i}$ and $\phi_{d}$ are coefficient, usually larger than 1, which is used to balance output values from proportion neurons, integration neurons and differentiation neurons. Output layer have $n$ neurons. The output of each neuron in this layer at $k$ is

$$
u_{p}(k)=\text { out }_{p}^{3}(k)=\sum_{l=1}^{n} \sum_{j=1}^{3} \omega_{j p}(k-1) o u t_{q j}^{2}(k-1)
$$

where $q$ is the number of subnets, that is, the number of output values. And $j$ is the number of neurons in hidden layer, $\omega_{i j}$ is weight between input layer and hidden layer, $\omega_{j k}$ is weight between hidden layer and output layer.

\subsection{Parameters initiation}

PSO algorithm searches for the optimal solution by collaboration among individuals in the population [25, 26]. In the algorithm, weight initiation is done randomly. However, weights may fall into local optima during the process of optimization. In this paper, particle swarm optimization (PSO) algorithm is adopted to set initial weights in the controller. The main steps of PSO algorithm are showed in Algorithm 2. 


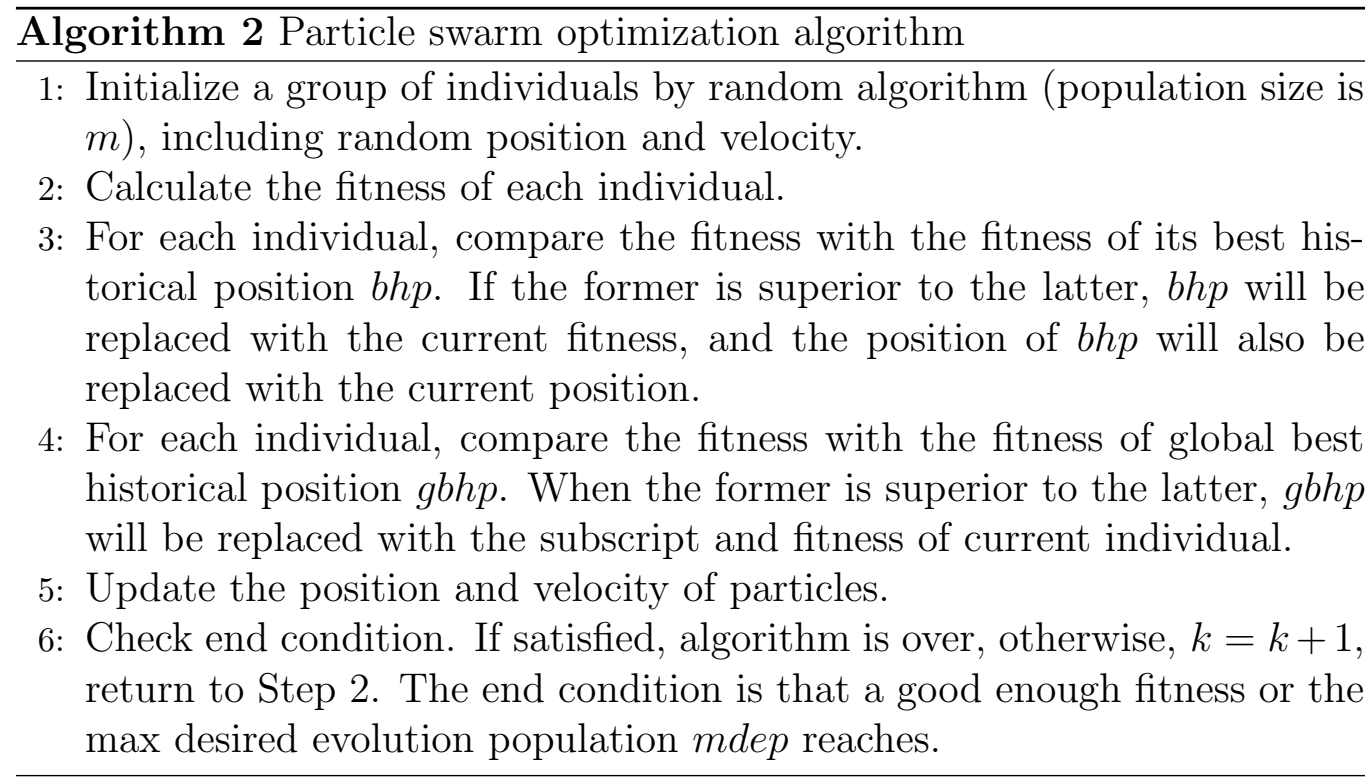

In Step 5, the position and velocity of particles are updated according to Equ. (7) and Equ. (8).

$$
\begin{gathered}
v_{i d}^{k+1}=v_{i d}^{k}+a \psi_{1}\left(p_{i d}^{k}-x_{i d}^{k}\right)+b \psi_{2}\left(p_{g d}^{k}-x_{i d}^{k}\right) \\
x_{i d}^{k+1}=x_{i d}^{k}+v_{i d}^{k+1}
\end{gathered}
$$

where $d \in[1,2, \cdots, n], i \in[1,2, \cdots, m], k$ is current evolution population, $\psi_{1}$ and $\psi_{2}$ are random number between 0 and $1, a$ and $b$ are acceleration constants. In order to prevent velocity of individual against great change, a max velocity is limited to a maximum of $V_{\max }$.

\subsection{Adaptive parameters adjustment}

Usually to get better control effect and close quickly to control object values, weights must be adjusted according to the error. The gradient descent method can be used to adjust the velocity. For function $f(X), X=$ $\left(x_{1}, x_{2}, \cdots, x_{n}\right)$, the gradient is shown in Equation (9).

$$
\operatorname{grad} f(X)=\left[\frac{\partial f(X)}{\partial x_{1}}, \frac{\partial f(X)}{\partial x_{2}}, \cdots, \frac{\partial f(X)}{\partial x_{n}}\right]^{T}
$$


Here the minus gradient direction is steepest descent direction. In this paper, an improved gradient descent method is designed considering the PID neural network. Gradient information is added into individual velocity with certain probability, which help particle to search the solutions more efficiently. The weights are changed by Equ. (10) and Equ. (11), which are presented as follows.

$$
\begin{aligned}
\omega_{i j}(k+1) & =\omega_{i j}(k)-\mu \frac{\partial e(k)}{\partial \omega_{i j}(k)}+\sigma\left[\omega_{i j}(k)-\omega_{i j}(k-1)\right] \\
\omega_{j p}(k+1) & =\omega_{j p}(k)-\mu \frac{\partial e(k)}{\partial \omega_{j p}(k)}+\sigma\left[\omega_{j p}(k)-\omega_{j p}(k-1)\right]
\end{aligned}
$$

where $\mu$ and $\sigma$ are the network learning rates, and $e(k)$ is control error calculated by Equ. (12).

$$
e(k)=\frac{1}{2} \sum_{q=1}^{n}\left|y_{q}^{*}(k)-x_{q}(k)\right|^{2}
$$

In our neural network, the parameters are computed in each sampling period. The weights are automatically adjusted on line based on the errors

of closed loop system, and the controller implements nonlinear and adaptive real-time online control for controlled system.

\section{Stability Analysis}

Let Lyapunov function be

$$
V(k)=\frac{1}{2} \sum_{q=1}^{n} e_{0}^{2}(k)
$$

where

$$
e_{0}(k)=y_{q}^{*}(k)-x_{q}(k)
$$

The change of Lyapunov function is

$$
\Delta V(k)=V(k+1)-V(k)=\frac{1}{2} \sum_{q=1}^{n}\left(\left(e_{0}^{2}(k+1)-e_{0}^{2}(k)\right)\right)
$$

That is 


$$
\begin{aligned}
\Delta V(k) & =\frac{1}{2} \sum_{q=1}^{n}\left(\left(e_{0}(k+1)-e_{0}(k)\right)\right)\left(e_{0}(k+1)+e_{0}(k)\right) \\
& =\frac{1}{2} \sum_{q=1}^{n} \Delta e_{0}(k)\left(2 e_{0}(k)+\Delta e_{0}(k)\right) \\
& =\sum_{q=1}^{n} e_{0}(k) \Delta e_{0}(k)+\frac{1}{2} \sum_{q=1}^{n} \Delta e_{0}^{2}(k)
\end{aligned}
$$

According to the Lyapunov method, when $\Delta V(k) \leq 0$ in any sampling period, the closed loop system is stable. That is,

$$
\sum_{q=1}^{n} e_{0}(k) \Delta e_{0}(k) \leq \frac{1}{2} \sum_{q=1}^{n} \Delta e_{0}^{2}(k)
$$

Based on Equ. (10) to Equ. (12), we can obtain Equ. (18).

$$
\begin{aligned}
\Delta \omega_{i j}(k) & =-\frac{\mu}{1-\sigma} \frac{\partial e(k)}{\partial \omega_{i j}(k)} \\
& \approx-\frac{\mu}{1-\sigma} e_{0}(k)\left(\sum_{q=1}^{n} e_{0}(k) \operatorname{sgn}\left(\frac{\Delta y_{q}^{*}(k)}{\Delta x_{q}(k)}\right)\right)
\end{aligned}
$$

Then

$$
\begin{aligned}
\Delta e_{0}(k) & =\sum_{q=1}^{n}\left(\sum_{i=1}^{3}\left(\frac{\partial e_{0}(k)}{\partial \omega_{i j}(k)} \Delta \omega_{i j}(k)\right)\right) \\
& =-\frac{\mu}{1-\sigma}\left(\sum_{i=1}^{3} \frac{1}{\omega_{i j}(k)}\right) \sum_{q=1}^{n}\left(e_{0}(k) \operatorname{sgn}\left(\frac{\Delta y_{q}(k)}{\Delta x_{q}(k)}\right)\right) \sum_{q=1}^{n} e_{0}^{2}(k)
\end{aligned}
$$

Let $h(k)=\sum_{q=1}^{n}\left(e_{0}(k) \operatorname{sgn}\left(\frac{\Delta y_{q}(k)}{\Delta x_{q}(k)}\right)\right) \sum_{q=1}^{n} e_{0}^{2}(k)$, Equ. (19) is simplified to

$$
\begin{aligned}
\Delta e_{0}(k) & =\sum_{q=1}^{n}\left(\sum_{i=1}^{3}\left(\frac{\partial e_{0}(k)}{\partial \omega_{i j}(k)} \Delta \omega_{i j}(k)\right)\right) \\
= & -\frac{\mu}{1-\sigma}\left(\sum_{i=1}^{3} \frac{1}{\omega_{i j}(k)}\right) h(k)
\end{aligned}
$$

Substitute to Equ. (17), then

$$
-\frac{\mu}{1-\sigma} h(k) \sum_{q=1}^{n} e_{0}(k)\left(\sum_{i=1}^{3} \frac{1}{\omega_{i j}(k)}\right) \leq \frac{1}{2}\left(\frac{\mu}{1-\sigma}\right)^{2} h^{2}(k) \sum_{q=1}^{n}\left(\sum_{i=1}^{3} \frac{1}{\omega_{i j}(k)}\right)^{2}
$$


To ensure $\Delta V(k) \leq 0$,

When $\left(\sum_{q=1}^{n} \sum_{i=1}^{3} \frac{e_{0}(k)}{\omega_{i j}(k)}\right) h(k)<0$, then

$$
0<\frac{\mu}{\sigma-1} \leq-2 \frac{\sum_{q=1}^{n} \sum_{i=1}^{3} \frac{e_{0}(k)}{\omega_{i j}(k)}}{\left(\sum_{q=1}^{n}\left(\sum_{i=1}^{3} \frac{1}{\omega_{i j}(k)}\right)^{2}\right) h(k)}
$$

When $\left(\sum_{q=1}^{n} \sum_{i=1}^{3} \frac{e_{0}(k)}{\omega_{i j}(k)}\right) h(k) \geq 0$, then

$$
-2 \frac{\sum_{q=1}^{n} \sum_{i=1}^{3} \frac{e_{0}(k)}{\omega_{i j}(k)}}{\left(\sum_{q=1}^{n}\left(\sum_{i=1}^{3} \frac{1}{\omega_{i j}(k)}\right)^{2}\right) h(k)} \leq \frac{\mu}{\sigma-1} \leq 0
$$

\section{Simulation}

A simulation is carried out to verify the proposed control strategy in this section. The controlled system is a complex nonlinear MIMO system with strong coupling of variables, described by Equ. 24).

$$
\left\{\begin{array}{l}
y_{1}(k+1)=0.4 \times y_{1}(k)+0.3 \times y_{2}(k)+\frac{u_{1}(k)}{1+u_{1}(k)^{2}}+0.2 \times u_{2}(k)^{3} \\
y_{2}(k+1)=0.3 \times y_{2}(k)+0.2 \times y_{3}(k)+\frac{u_{2}(k)}{1+u_{2}(k)}+0.6 \times u_{1}(k)^{3} \\
y_{3}(k+1)=0.5 \times y_{3}(k)+0.3 \times y_{1}(k)+\frac{u_{3}(k)}{1+u_{3}(k)^{2}}+0.1 \times u_{2}(k)^{3}
\end{array}\right.
$$

Where $u_{1}, u_{2}, u_{3}$ are control laws. The following parameters are set to the system:

- Initial values of control system is specified as $\left[\begin{array}{lll}0 & 0 & 0\end{array}\right]$.

- Object values of control system is specified as $\left[\begin{array}{lll}0.7 & 0.4 & 0.8\end{array}\right]$.

- Learning rates is specified as 0.006 .

- Time interval is specified as 0.0001s. 


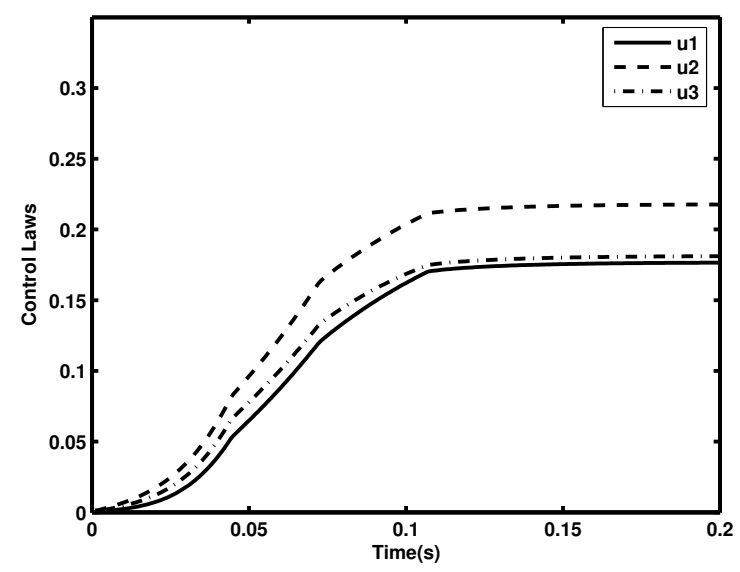

Figure 3: Control laws vary with time.

During the process of weights initiation by PSO, numbers of populations is specified as 50, and iteratiion number is specified as 40. To illustrate the advantages of our controller, three different methods are used to control the same system separately.

\subsection{Control by traditional PID neutral network}

The first simulation is controlling the system by traditional PID neutral network, and the results are shown from Figure 3 to Figure 5 . Figure 3 shows the control laws changed with time. Figure 4 shows the contrast between the actual output values and the object output values. Figure 5 shows control error with time variation.

Simulation results show that the actual output is close to expect output, control law is gradually stabilized, and control error is close to 0. That is to say, this method has some effect on control the system.

\subsection{Control by PID neutral network though standard PSO optimization}

The second simulation is controlling the same system by PID neutral network which is optimized by standard PSO, and the results are shown from Figure 6 to Figure 8. Figure 6 shows the control laws changed with time. Figure 7 depicts the contrast between the actual output values and the object output values. Figure 8 illustrates the control error with time variation. 

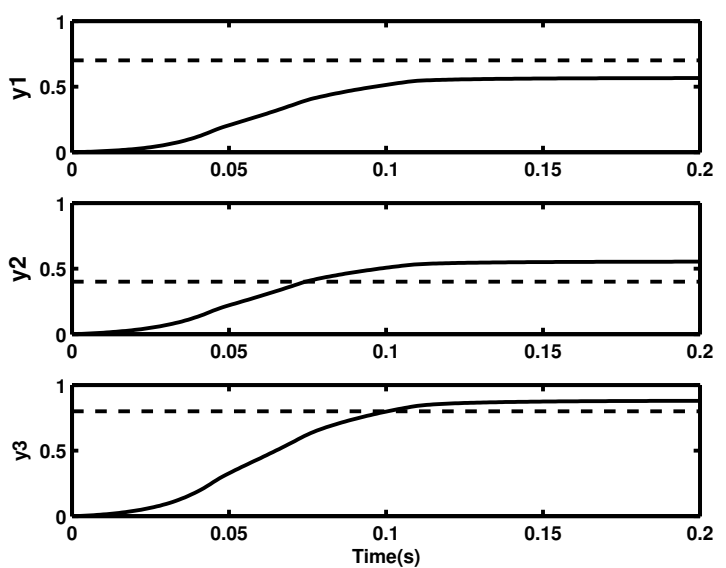

Figure 4: Actual output values vary with time.

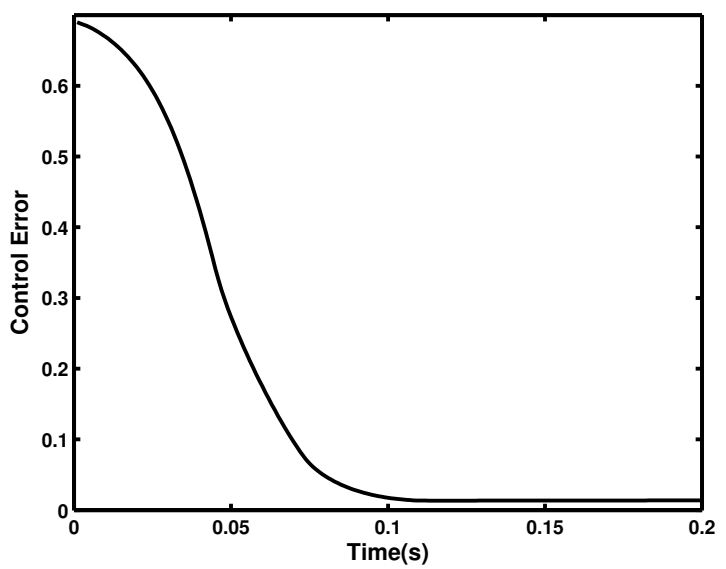

Figure 5: Control error vary with time. 


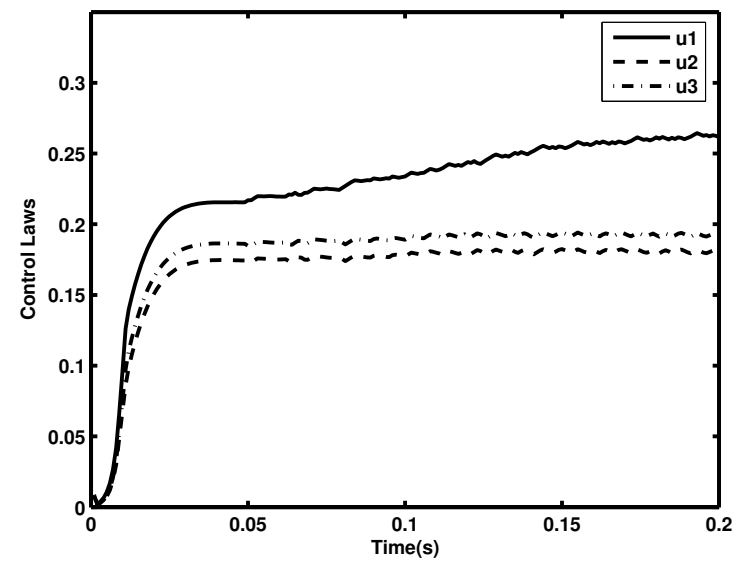

Figure 6: Control laws vary with time.
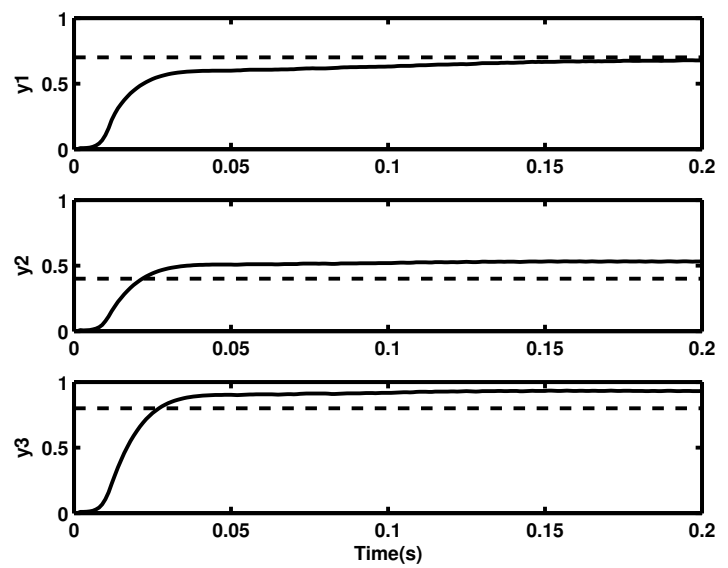

Figure 7: Actual output values vary with time. 


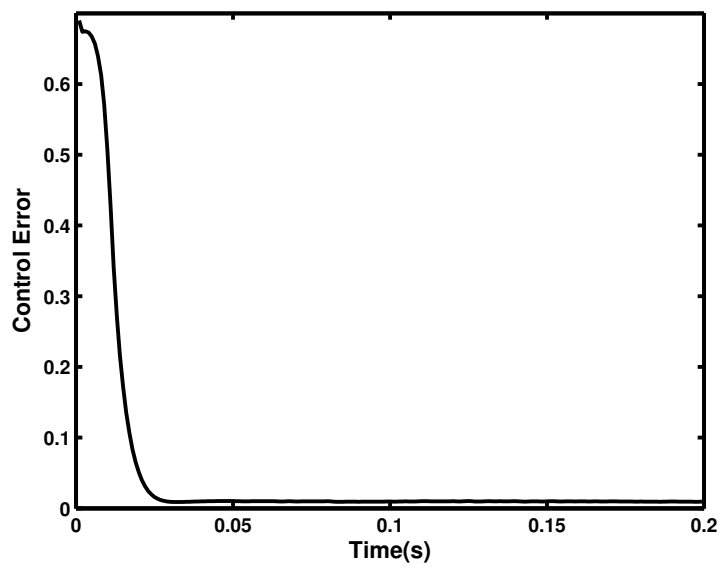

Figure 8: Control error vary with time.

Simulation results show that the actual output is close to the expected output, and the speed of convergence is faster than the previous method and the control law is gradually stabilized.

\subsection{Control by the adaptive PID neutral network}

The last simulation is controlling the same system by adaptive PID neutral network, which is proposed in this paper, and the results are shown from Figure 9 to Figure 11. Figure 9 shows the control laws changed with time. Figure 10 shows the contrast between the actual output values and the object output values. Figure 11 shows control error with time variation.

In order to compare the performance of three different control methods mentioned above, the control errors varied with time are shown respectively in Table 1. Due to the limit of space, the time interval of data is $0.001 \mathrm{~s}$, and 15 data groups is selected from 0.02s. Obviously, adopting the new method, the actual output values can most quickly approximate the object output values when compared with the previous two methods. The control error is falling faster before $0.02 \mathrm{~s}$, then tends to 0 gradually. The control laws are also quickly changed to constant within a short time. Therefore, the adaptive PID neutral network has high convergence speed, high accuracy and high stability for the control of a complex nonlinear system. 


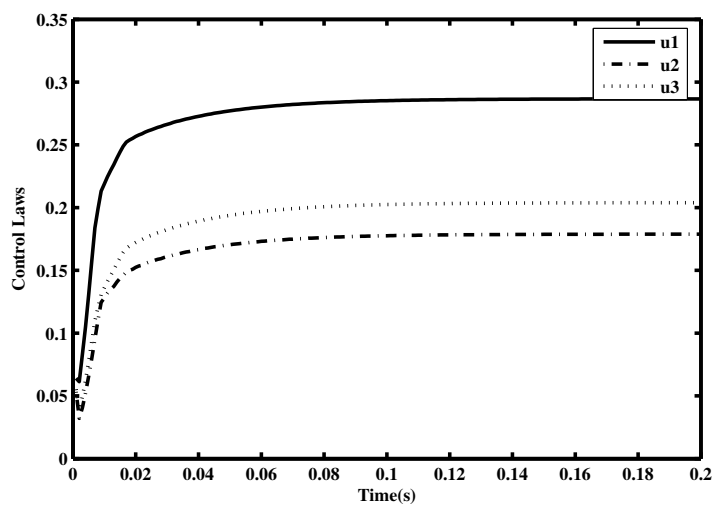

Figure 9: Control laws vary with time.
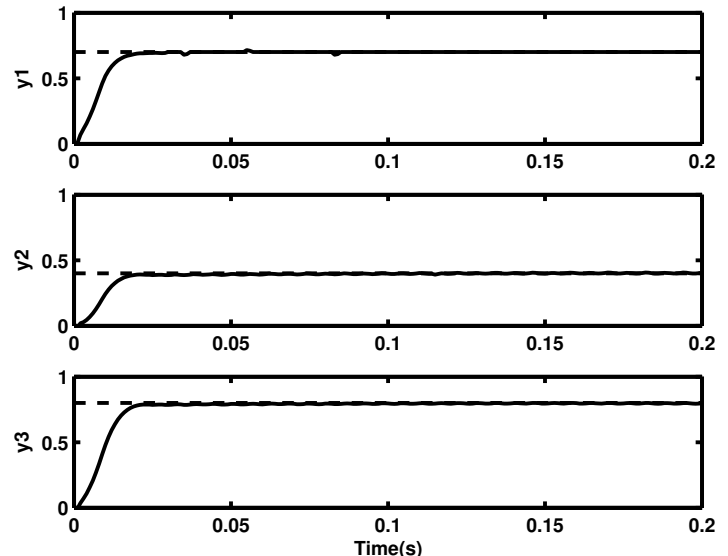

Figure 10: Actual output values vary with time. 


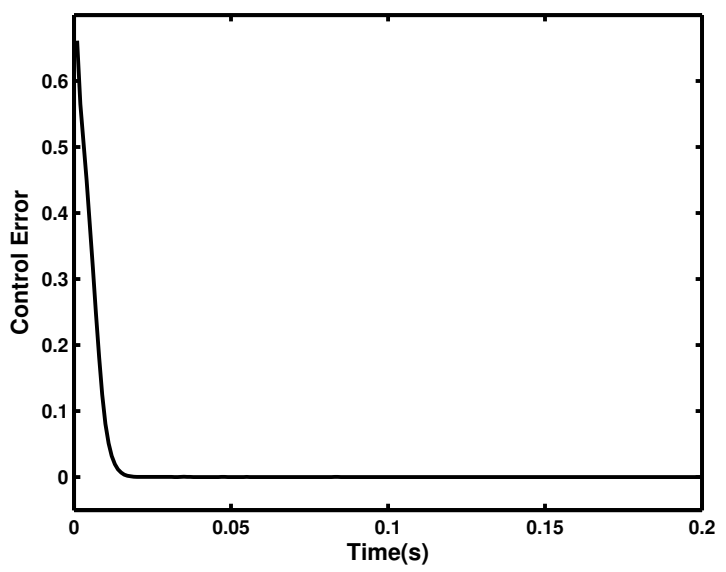

Figure 11: Control error vary with time.

Table 1: Control error vary with time using above three control methods.

\begin{tabular}{|c|c|c|c|c|}
\hline Groups & Times & Control Error I a & Control Error II b & Control Error III ${ }^{\circ}$ \\
\hline 1 & 0.020 & 0.626280 & 0.050725 & 0.000322 \\
\hline 2 & 0.021 & 0.620422 & 0.039564 & 0.000204 \\
\hline 3 & 0.022 & 0.614219 & 0.030990 & 0.000174 \\
\hline 4 & 0.023 & 0.607665 & 0.024495 & 0.000165 \\
\hline 5 & 0.024 & 0.600709 & 0.019648 & 0.000168 \\
\hline 6 & 0.025 & 0.593351 & 0.016086 & 0.000221 \\
\hline 7 & 0.026 & 0.585584 & 0.013522 & 0.000194 \\
\hline 8 & 0.027 & 0.577358 & 0.011724 & 0.000158 \\
\hline 9 & 0.028 & 0.568675 & 0.010503 & 0.000185 \\
\hline 10 & 0.029 & 0.559526 & 0.009712 & 0.000234 \\
\hline 11 & 0.030 & 0.549869 & 0.009237 & 0.000214 \\
\hline 12 & 0.031 & 0.539705 & 0.008988 & 0.000165 \\
\hline 13 & 0.032 & 0.529032 & 0.008897 & 0.000103 \\
\hline 14 & 0.033 & 0.517811 & 0.008912 & 0.000103 \\
\hline 15 & 0.034 & 0.506049 & 0.008997 & 0.000138 \\
\hline Mean & - & 0.573084 & 0.018133 & 0.000183 \\
\hline
\end{tabular}

${ }^{\mathrm{a}}$ Control Error by 4.1.

${ }^{\mathrm{b}}$ Control Error by 4.2 .

${ }^{\mathrm{c}}$ Control Error by 4.3 . 


\section{Conclusion}

In this paper, the design of an adaptive PID Neural Network controller is presented. The controller's model is established based on a PID Neural network. The PSO algorithm is adopted to select initial weights, solving the problem that influences the initial values in the training, improving the convergent speed, and preventing the weights getting trapped into local optima. In each sampling period, improved gradient descent method is used to change all weights in this network. With three main features such as self-correcting, on-line and real-time, the adaptive mechanism of parameters adjustment can compensate the drawbacks of the conventional methods. The stability is analyzed according to the Lyapunov method.

Empirical results illustrate that the adaptive PID Neural Network controller is significantly better than the traditional PID neutral network controller and PID neutral network optimized using PSO. Our controller can achieve better control results within less sampling periods and the error tends to 0 in a stable manner. During the weight initialization, PSO algorithm takes a long time and this requires more research and also to decide the number of iterations to have a nice balance between high efficiency and precision. The proposed control approach is available to some systems with complex nonlinear characteristics and it could also be extended to other nonlinear systems in natural and social sciences.

\section{Acknowledgment}

This work is supported partly by the National Natural Science Foundation of China (Grant No. 51075289, 61173035), the Shanxi Natural Science Foundation (Grant No. 2011011019-3), the project GACR P202/10/0262, the IT4Innovations Centre of Excellence project, reg. no. CZ.1.05/1.1.00/02.0070 supported by Operational Programme 'Research and Development for Innovations' funded by Structural Funds of the European Union and state budget of the Czech Republic and by long-term strategic development financing of the Institute of Computer Science (RVO: 67985807).

\section{References}

[1] A. Boulkroune, M. Tadjine, M. M'Saad, M. Farza, Fuzzy adaptive controller for mimo nonlinear systems with known and unknown control direction, Fuzzy Sets and Systems 161 (6) (2010) 797-820. 
[2] N. Reza, R. Ahmad, Adaptive decoupled control of 4-leg voltage-source inverters for standalone photovoltaic systems: Adjusting transient state response, Renewable Energy 36 (10) (2011) 2733-2741.

[3] J. Liu, W. Wang, F. Golnaraghi, E. Kubica, Fuzzy adaptive observer backstepping control for mimo nonlinear systems, Fuzzy Sets and Systems 160 (21) (2010) 2746-2759.

[4] Y. Wang, L. Nie, Application of fuzzy control on smart car servo steering system, Advanced Science Letters 4 (6-7) (2011) 2099-2103.

[5] D. Rastovic, Tokamak design as one sustainable system, Neural Network World 21 (6) (2011) 493-504.

[6] M. Chen, C. Jiang, Q. Wu, Disturbance-observer-based robust flight control for hypersonic vehicles using neural networks, Advanced Science Letters 4 (4-5) (2011) 1771-1775.

[7] V. Akpan, G. Hassapis, Nonlinear model identification and adaptive model predictive control using neural networks, ISA Transactions 50 (2) (2011) 177-194.

[8] E. Irigoyen, M. Larrea, J. Valera, V. Gómez, F. Artaza, A hybridized neuro-genetic solution for controlling industrial r3 workspace, Neural Network World 20 (7) (2010) 811-824.

[9] J. Garrido, F. Vzquez, F. Morilla, Centralized multivariable control by simplified decoupling, Journal of Process Control 22 (6) (2012) 10441062.

[10] C. Cha, S. Kim, L. Cao, H. Kong, Decoupled control of stiffness and permeability with a cell-encapsulating poly(ethylene glycol) dimethacrylate hydrogel, Biomaterials 31 (18) (2010) 4864-4871.

[11] I. García-Herreros, X. Kestelyn, J. Gomand, R. Coleman, P. Barre, Model-based decoupling control method for dual-drive gantry stages: A case study with experimental validations, Control Engineering Practice 21 (3) (2013) 298-307.

[12] A. Nassirharand, Computer-aided Nonlinear Control System Design, Springer Verlag, 2012. 
[13] L. Grigsby, Power System Stability and Control, Third Edition, CRC Press, 2012.

[14] A. Veloni, A. Palamides, Control System Problems: Formulas, Solutions, and Simulation Tools, CRC Press, 2012.

[15] H. Jafarnejadsani, J. Pieper, J. Ehlers, Adaptive control of a variablespeed variable-pitch wind turbine using radial-basis function neural network, IEEE Transactions on Control Systems Technology (2013) Online.

[16] Y.-Y. Lin, J.-Y. Chang, C.-T. Lin, Identification and prediction of dynamic systems using an interactively recurrent self-evolving fuzzy neural network, IEEE Transactions on Neural Networks and Learning Systems 24 (2) (2013) 310-321.

[17] M. Chemachema, Output feedback direct adaptive neural network control for uncertain siso nonlinear systems using a fuzzy estimator of the control error, Neural Networks 26 (2012) 25-34.

[18] A. Abraham, H. Guo, H. Liu, Swarm intelligence: foundations, perspectives and applications, Swarm Intelligent Systems (2006) 3-25.

[19] Y.-S. Yang, W.-D. Chang, T.-L. Liao, Volterra system-based neural network modeling by particle swarm optimization approach, Neurocomputing 82 (2012) 179-185.

[20] S. Selvakumaran, S. Parthasarathy, R. Karthigaivel, V. Rajasekaran, Optimal decentralized load frequency control in a parallel ac-dc interconnected power system through hvdc link using pso algorithm, Energy Procedia 14 (2012) 1849-1854.

[21] A. Hasni, B. Draoui, M. Latfaoui, T. Boulard, Identification of natural ventilation parameters in a greenhouse with continuous roof vents, using a pso and gas, Sensors and Transducers 119 (8) (2010) 182-192.

[22] D. Chen, F. Zou, J. Wang, A multi-objective endocrine pso algorithm and application, Applied Soft Computing 11 (8) (2011) 4508-4520.

[23] A. Boulkroune, M. M'Saad, M. Farza, Adaptive fuzzy tracking control for a class of mimo nonaffine uncertain systems, Neurocomputing 93 (15) (2012) 48-55. 
[24] H. Wang, B. Chen, C. Lin, Adaptive neural control for strict-feedback stochastic nonlinear systems with time-delay, Neurocomputing 77 (1) (2012) 267-274.

[25] D. Chen, C. Zhao, Particle swarm optimization with adaptive population size and its application, Applied Soft Computing 9 (1) (2009) 39-48.

[26] S. Klein, J. Pluim, M. Staring, M. Viergever, Adaptive stochastic gradient descent optimisation for image registration, International Journal of Computer Vision 81 (3) (2009) 227-239.

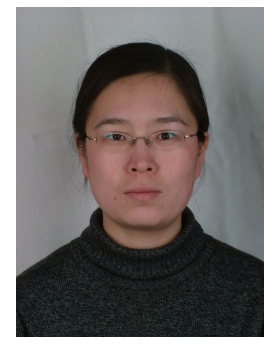

Jun Kang received her B.Eng. degree in computer and its application in 2000, M.Eng. degree in artificial intelligence in 2005, both from North University of China (NUC), China. Currently, she is a Ph.D. candidate at Taiyuan University of Science and Technology (TYUST), China. Her research interests include mechanical and electrical system control, and artificial intelligence.

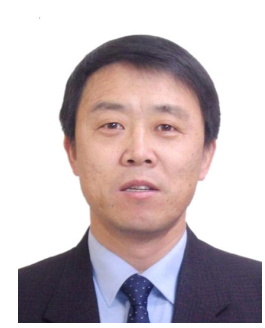

Wenjun Meng received his B.Eng. degree in lifting and conveying mechanism in 1984 and M.Eng. degree in construction mechanism in 1990, both from Taiyuan University of Science and Technology (TYUST), China. He received his $\mathrm{Ph} . \mathrm{D}$. degree in mechanical and electronics engineering from Beijing University of Aeronautics \& Astronautics), China, in 2005. Currently, he is a professor and dean with the School of Mechanical Engineering, TYUST. His research interests include all aspects of Study and Control for Mechanical and Electrical System, the fundamentals and industrial applications of solids handling and storage systems including 
mechanical conveying, hoppers and control, material quality, characterization and instrumentation, and etc.

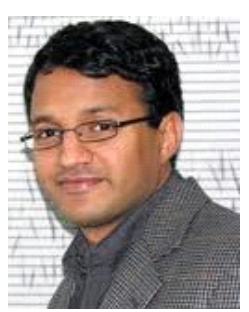

Ajith Abraham is the Director of Machine Intelligence Research Labs (MIR Labs), Scientific Network for Innovation and Research Excellence, WA, USA, with a joint appointment in the IT For Innovations - Center of excellence at VSB - Technical University of Ostrava, Czech Republic. He also holds an Adjunct Professor appointment in Dalian Maritime University, China. He received the Ph.D. degree in Computer Science from Monash University, Melbourne, Australia. His research and development experience includes more than 23 years in the industry and academia. He works in a multidisciplinary environment involving machine intelligence, network security, various aspects of networks, e-commerce, Web intelligence, Web services, computational grids, data mining and their applications to various real-world problems. He has authored/co-authored more than 900 publications (h-index $=50+$ ), and some of the works have also won best paper awards at international conferences. He has given more than 60 plenary lectures and conference tutorials in these areas. He serves/has served the editorial board of over 50 International journals and has also guest edited over 40 special issues on various topics. Since 2008, he is the Chair of IEEE Systems Man and Cybernetics Society Technical Committee on Soft Computing and a Distinguished Lecturer of IEEE Computer Society representing Europe (since 2011). More information at: http://www.softcomputing.net. 


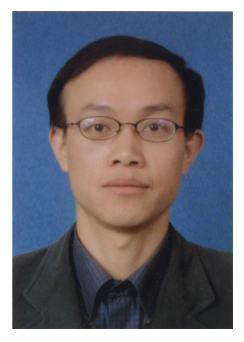

Hongbo Liu received his three level educations (B. Sc., M. Sc., Ph.D.) at the Dalian University of Technology, China. Currently he is a professor at School of Information Engineering and Science, Dalian Maritime University, with an affiliate appointment in the Institute for Neural Computation, University of California San Diego, USA. His research interests are in system modeling and optimization involving soft computing, probabilistic modeling, cognitive computing, machine learning, data mining, etc. He participates and organizes actively international conference and workshop and international journals/publications. 